\title{
Síndrome de burnout: fatores sociodemográficos e ocupacionais em profissionais da enfermagem
}

\author{
Burnout syndrome: sociodemographic and occupational factors in nursing professionals \\ Síndrome de burnout: factores sociodemográficos y ocupacionales en profesionales de \\ enfermería
}

Italo Everton Bezerra Barbosa ${ }^{1 *}$, Alicia Ribeiro Fonseca1 ${ }^{1}$ Emmanuele Neuza Moreira de Andrade ${ }^{1}$, Dandara Conceição Maklouf ${ }^{1}$, Meire Cristina Soares Ribeiro ${ }^{1}$, Antônio José Paulo da Silva Rodrigues ${ }^{1}$, Breno de Souza Mota ${ }^{2}$, Yasmin Tainá Corrêa Laborda ${ }^{1}$, Vitória Diniz Bezerra Lúcio da Silva', Felipe Chrystian de Figueiredo Lira'.

\section{RESUMO}

Objetivo: Avaliar a incidência da Síndrome de Burnout (SB) em enfermeiros, auxiliares e técnicos de enfermagem, visando determinar variáveis sociodemográficas e ocupacionais, a fim de proporcionar elementos para uma compreensão mais aprofundada da SB nesses profissionais. Métodos: Trata-se de uma pesquisa do tipo descritiva, com abordagem qualitativa, utilizando a revisão integrativa de literatura (RIL) como técnica. O levantamento de dados foi realizado entre os meses de outubro de 2020 e janeiro de 2021, no banco de dados da Scientific Electronic Library Online (SCIELO) e Biblioteca Virtual em Saúde (BVS), utilizando os descritores em saúde: "Síndrome de Burnout AND Profissionais da Saúde AND Enfermagem AND Trabalho", sendo encontrados 133 publicações na totalidade, após a aplicação dos critérios de elegibilidade e inelegibilidade, foram considerados 11 artigos para compor esse estudo. Resultados: $A$ prevalência da SB em profissionais de enfermagem ainda e bastante significativa, com maior incidência nos auxiliares de enfermagem (52,94\%), estando relacionado principalmente com a sobrecarga de trabalho. Considerações finais: Logo, torna-se perceptível a necessidade de as organizações lidarem com o estado de saúde dos funcionários, particularmente aquelas com maior proximidade físico-psicológica com o paciente/familiares, para que a SB possa ser prevenida.

Palavras-chave: Esgotamento psicológico, Enfermagem, Trabalho.

\begin{abstract}
Objective: To evaluate the incidence of Burnout Syndrome (BS) in nurses, nursing assistants and technicians, aiming to determine sociodemographic and occupational variables, in order to provide elements for a deeper understanding of BS in these professionals. Methods: This is a descriptive research, with a qualitative approach, using the integrative literature review (ILR) as the technique. The data survey was carried out between the months of October 2020 and January 2021, in the database of Scientific Electronic Library Online (SCIELO) and Virtual Health Library (VHL), using the health descriptors: "Burnout Syndrome AND Health Professionals AND Nursing AND Work ", being found 133 publications in total, after applying the eligibility and ineligibility criteria, 11 articles were considered to compose this study. Results: The prevalence of BS in nursing professionals is still quite significant, with a higher incidence in nursing assistants $(52.94 \%)$, being mainly related to work overload. Final considerations: Therefore, it becomes clear the need for organizations to deal with the health status of employees, particularly those with greater physical and psychological proximity to the patient / family, so that BS can be prevented.
\end{abstract}

Keywords: Psychological burnout, Nursing, Work.

${ }^{1}$ Centro Universitário FAMETRO (CEUNI-FAMETRO), Manaus - AM. *E-mail: italoeverton1998@gmail.com 2Universidade de São Paulo (USP), São Paulo - SP. 


\section{RESUMEN}

Objetivo: Evaluar la incidencia del Síndrome de Burnout (SB) en enfermeros, auxiliares de enfermería y técnicos, con el objetivo de determinar variables sociodemográficas y ocupacionales, con el fin de brindar elementos para una comprensión más profundas del SB en estos profesionales. Métodos: Se trata de una investigación descriptiva, con enfoque cualitativo, utilizando como técnica la revisión integrativa de la literatura (EIR). La encuesta de datos se realizó entre los meses de octubre de 2020 y enero de 2021, en la base de datos de la Biblioteca Electrónica Científica Online (SCIELO) y Biblioteca Virtual en Salud (BVS), utilizando los descriptores de salud: "Síndrome de Burnout Y Profesionales de la Salud Y Enfermería Y Trabajo ", encontrándose 133 publicaciones en total, luego de aplicar los criterios de elegibilidad e inelegibilidad, se consideraron 11 artículos para componer este estudio. Resultados: La prevalencia de SB en profesionales de enfermería sigue siendo bastante significativa, con una mayor incidencia en auxiliares de enfermería $(52,94 \%)$, principalmente relacionada con la sobrecarga de trabajo. Consideraciones finales: Por lo tanto, se hace evidente la necesidad de que las organizaciones se ocupen del estado de salud de los empleados, en particular de aquellos con mayor proximidad física y psicológica al paciente / familia, de manera que se pueda prevenir el BS.

Palabras clave: Agotamiento psicológico, Enfermería, Trabajo.

\section{INTRODUÇÃO}

Servir no ambiente hospitalar implica tanto em tarefas relaxantes quanto operações estressantes. Grande parte dessas práticas estressantes cabe aos profissionais de saúde, auxiliares, assistentes e clínicos, pois são responsáveis pelo tratamento primário de pacientes doentes e, portanto, sustentam a proximidade com o desconforto e o sofrimento dos outros. No entanto, devido à baixa remuneração, muitos trabalhadores de enfermagem sucumbem ao trabalho vindo a dobrarem as horas dentro do âmbito hospitalar e em última instância, cedem a vários fatores particulares de sua vida social em prol do trabalho, afetando em um grave sofrimento emocional (MOTA BS, et al., 2020).

A experiência dicotômica das emoções encontradas pelos profissionais de enfermagem na área do emprego mostra que, à medida que alguns estudiosos postulam, o trabalho pode ser uma fonte de realização ou decepção pessoal, a partir de uma variedade de influências individuais e ocupacionais. Se prevalecer para o lado negativo, o significado que o trabalho pode ter para o trabalhador acaba sendo perdido, e seu funcionamento mental é diretamente influenciado por isso (VIEIRA I e RUSSO J, 2019).

O trabalho é o principal mediador da realização social do indivíduo contemporâneo e atualmente não há nada que possa substituí-lo nessa posição. Nas últimas décadas, o clima de trabalho tem experimentado metamorfoses dramáticas. Essa questão é vista como a ponta do iceberg de um problema mais complicado, no qual se observa a precariedade dos ambientes de trabalho e parcerias. Assim, a angústia a que o tema está ligado no mundo do emprego acaba sendo um elemento extremamente significativo na avaliação de transtornos relacionados ao estresse (OLIVEIRA RF, et al., 2017).

O estresse por si só pode ser entendido como uma condição marcada por mudanças fisiológicas e psicológicas, processadas no corpo enquanto está em uma circunstância que envolve uma resposta mais extrema do que aquela que leva ao seu funcionamento habitual, de acordo com o conceito mais utilizado na literatura científica. Esse conceito contribuiu para o desenvolvimento de um modelo de tensão trifásica, que envolve os estágios de alerta, resistência e fadiga. Essas etapas emergem diante da inadequação dos esforços adaptativos mobilizados pela ocorrência traumática, para o autor em questão, podendo levar a pessoa ao seu estado máximo (MOURÃO AL, et al., 2017).

Herbert Freudenberger (1974), cunhou o termo burnout em meio aos avanços conceituais do paradigma do estresse trifásico para caracterizar uma síndrome em que a fadiga profissional era sintomatológica. Esta palavra é derivada do verbo em inglês "burnout", que significa "queimar inteiramente" ou "consumir", em português. Assim, Freudenberger definiu a síndrome de burnout (SB) como uma sensação de perda e fadiga 
devido ao desgaste desnecessário de energia e recursos internos. Além disso, exaustão, irritabilidade, depressão, rigidez e inflexibilidade desempenharam um papel importante na composição da síndrome, onde tais fatores foram descobertos mais tarde pelo o mesmo (VIEIRA I e RUSSO J, 2019).

$\mathrm{Na}$ década seguinte, em comparação com a perspectiva psiquiátrica de Freudenberger, Maslach e Jackson (1971), colocaram o burnout no sentido das relações entre traços humanos e especificidades de definição de trabalho. A condição foi então entendida como um mecanismo desencadeado pela experiência ocupacional de quantidades severas e sustentadas de estresse. Além disso, vale ressaltar que burnout se define como uma condição tridimensional, consistindo nos seguintes fatores: fadiga mental, despersonalização e frustração ocupacional, para fortalecer esse ponto de vista psicossocial (SILVA DSD, et al., 2015).

A fadiga emocional é definida por uma perda de energia que inclui sentimentos de desesperança, isolamento, tristeza, frustração, impaciência, irritabilidade, estresse, empatia reduzida, bem como aumento da vulnerabilidade a doenças como cefaleia, náusea, desconforto lombar ou cervical, tensão muscular e distúrbios do sono. A despersonalização, por outro lado, é vista como artefatos inanimados através do cuidado de pacientes, pares e organização, desencadeando a sensação de isolamento em contraste com os outros. Finalmente, a decepção profissional é um padrão comportamental moldado por uma inclinação pessimista para se julgar, fazendo com que o profissional se sinta insatisfeito com o sucesso de seu trabalho (RAMOS CEB, et al., 2019).

A presença de grandes diferenciações deve ser mencionada, com exceção das aproximações lógicas que podem ser delineadas entre o estresse, em especial o chamado estresse ocupacional, ou seja, o estresse decorrente da função, e o burnout. O engajamento de parcerias íntimas com os trabalhadores do setor de trabalho é ilustrado na definição de burnout, pois contribui para uma perda substancial da prestação de serviços. Há a hipótese de que profissionais de ambos os segmentos sejam suscetíveis ao estresse ocupacional, enquanto apenas profissionais baseados especificamente no tratamento do outro são suscetíveis ao crescimento de burnout. Os profissionais de enfermagem devem ser considerados entre os profissionais concentrando-se principalmente no tratamento de outros em uma posição de considerável suscetibilidade a SB (DUTRA HS, et al., 2019)

Afinal, por causa da ideologia humanista que a caracteriza e do fato de enfrentarem no mercado de trabalho, a divergência entre as aspirações que têm em relação ao trabalho desencadeará sofrimento emocional significativo. Além disso, a maioria dos profissionais de enfermagem estabelece suas práticas no sentido hospitalar, de tal forma que estão sujeitos a estressores no local de trabalho que prejudicam seu bemestar mental, como longas horas de trabalho, instalações insuficientes e vulnerabilidade a riscos químicos e físicos (SILVA-JUNIOR RF, et al., 2020).

É necessário ressaltar que os profissionais de enfermagem estão tentando aceitar sua verdadeira posição na comunidade, a fim de alcançar mais respeito e melhor remuneração. Também encontraram desafios nesta fase que podem comprometer o exercício de suas operações devido à desmotivação, tendo consequências emocionais. Sua identidade pessoal pode ser comprometida e, como resultado, impactar seu engajamento relacionado ao trabalho. Assim, gera-se uma situação que contribui para o desenvolvimento de atitudes depreciativas contra pacientes, colegas de trabalho e a organização do hospital, o que caracteriza o fenômeno do burnout em seus aspectos (FERNANDE SLS, et al., 2018).

O valor de dedicar maior consideração ao burnout em praticantes de enfermagem e suas causas relevantes parece inquestionável à luz do acima. No cenário externo, com esse método, muitos estudiosos estabeleceram estudos metodológicos, conforme revelado por análises bibliográficas. No entanto, ainda há muitos poucos estudos nacionais que têm esse caráter e apresentam esse conceito. É preciso reverter essa situação para introduzir, com base em provas empíricas, políticas públicas capazes de evitar que os efeitos negativos do burnout atinjam níveis cada vez mais preocupantes no país no nível governamental, social e pessoal (OLIVEIRA RF, et al., 2017). 
Todavia, o presente estudo teve como objetivo criar algumas associações entre a incidência da SB em enfermeiros, auxiliares e técnicos de enfermagem, visando determinar variáveis sociodemográficas e ocupacionais, a fim de proporcionar elementos para uma compreensão mais aprofundada da SB nos profissionais de enfermagem.

\section{MÉTODOS}

A metodologia aplicada na presente pesquisa documental foi estabelecida através de coleta de dados, no qual foi realizada a partir de consultas com informações de arquivos. Este desenho conceitual é consistente com a ênfase da ciência naturalista, uma vez que o objetivo principal da pesquisa experimental agrupada sob este título não é avaliar teorias previamente desenvolvidas, mas sim explicar um fenômeno como acontece normalmente e em condições que a produzem espontaneamente (SOUSA LMM, et al., 2017).

Trata-se de uma pesquisa descritiva, qualitativa, utilizando a revisão integrativa de literatura (RIL) como técnica, realizada entre os meses de outubro de 2020 e janeiro de 2021. A busca de dados foi feita por meio do acesso aos bancos de dados da Scientific Electronic Library Online (SCIELO) e Biblioteca Virtual em Saúde (BVS). As bases de dados foram escolhidas por conta de sua acessibilidade e atualizações em revistas. Apenas artigos realizados com humanos foram incluídos na análise com a permissão de um comitê de ética científica.

A análise integrativa é uma forma mais ampla de revisão, uma vez que torna possível utilizar a literatura analítica e científica com diversos métodos experimentais (quantitativo e qualitativo). Em referência aos seus objetivos, fontes e metodologia, os artigos utilizados no estudo são sistematicamente revisados, permitindo ao leitor examinar as informações preexistentes sobre o tema em análise. Este método de estudo tenta compilar e sintetizar, de forma ordenada, os achados da pesquisa sobre um tema específico, levando ao aprofundamento do assunto escolhido. A pesquisa foi desenvolvida em cinco fases: criação da problemática; coleta de dados; avaliação de artigos; revisão e avaliação de dados e apresentação de achados (SOARES CB, et al., 2014).

Diante do que descreve na literatura e com base no que foi lido a seguinte questão do estudo foi formatada para a realização do processo de investigação, levando em consideração às leituras, debates e conversas sobre a SB, vindo a forma a seguinte pergunta norteadora: Quais são os principais fatores sociodemográficos e ocupacionais da SB, e como a mesma possui influências sobre os profissionais de enfermagem?

Para a obtenção dos materiais adquiridos para a pesquisa, foram pesquisadas tais palavras-chaves como, Síndrome de Burnout AND Profissionais da Saúde AND Enfermagem AND Trabalho. Os critérios de elegibilidade foram: publicações gratuitas, disponíveis na íntegra, nos idiomas português e inglês, publicados no período de 2015 a 2020. Os critérios de inelegibilidade foram: publicações indisponíveis de forma gratuita, publicados nos anos anteriores ao ano de 2015 , fora dos idiomas português e inglês, e que não estavam envolvidos com base no tema da pesquisa.

\section{RESULTADOS}

Utilizando os descritores "Síndrome de Burnout AND Profissionais da Saúde AND Enfermagem AND Trabalho" foram encontradas 133 publicações no total, 22 presentes no banco de dados da SCIELO e 111 no BVS, todos os artigos estavam disponíveis na íntegra. Ao adicionar o filtro dos idiomas português e inglês, o número reduziu para 126; ao adicionar o critério de artigos publicados durante 2015 a 2020, o número diminuiu para 67. Após a seleção, leitura e análise dos artigos, foram selecionados 11 para compor esta revisão (Figura 1).

Dentre os tipos de artigos selecionados para revisão, sete são do tipo transversais $(63.5 \%)$, e quatro descritivos (36.5\%). Para melhor compreensão dos estudos selecionados para revisão integrativa, será apresentado no quadro (Quadro 1), uma síntese completa dos estudos, conforme o ano de publicação, autor, título, objetivo, estudo e publicação. 
Figura 1 - Fluxograma das etapas da seleção dos artigos para revisão.

Definição da pergunta norteadora:

Quais são os principais fatores sociodemográficos e ocupacionais da SB, e como a mesma possui influências sobre os profissionais de enfermagem?

Encontrados: 133 Artigos sem critérios. SCIELO: 22; BVS: 111

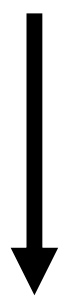

Após a aplicação dos critérios de elegibilidade e inelegibilidade: 67 artigos publicados direcionados ao tema.
Busca na Base de Dados por meio

do Scientific Electronic Library

Online (SCIELO) e Biblioteca Virtual em Saúde (BVS) com a seguinte combinação: Síndrome de Burnout AND Profissionais da Saúde AND Enfermagem AND Trabalho

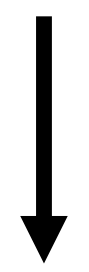

Descritores em ciências da saúde (DeCS): Síndrome de Burnout AND Profissionais da Saúde AND Enfermagem AND Trabalho.

Após a seleção, leitura e análise dos artigos, foram selecionados 11 artigos para revisão. SCIELO: 2; BVS: 9

Fonte: Barbosa IEB, et al., 2021. 
Quadro 1 - Artigos selecionados para revisão.

\begin{tabular}{|c|c|c|c|c|c|}
\hline $\mathbf{N}^{\circ}$ & Autor/Ano & Título & Objetivo & Estudo & Publicação \\
\hline 1 & Campos IC, et al. (2015) & $\begin{array}{l}\text { Fatores Sociodemográficos e } \\
\text { Ocupacionais Associados à Síndrome } \\
\text { de Burnout em Profissionais de } \\
\text { Enfermagem }\end{array}$ & $\begin{array}{l}\text { Investigar a prevalência da síndrome de Burnout nos } \\
\text { profissionais de enfermagem de um hospital e de } \\
\text { Unidades Básicas de Saúde, comparando-a entre as } \\
\text { três categorias profissionais (enfermeiros, técnicos e } \\
\text { auxiliares de enfermagem) }\end{array}$ & $\begin{array}{l}\text { Corte transversal com } \\
\text { abordagem quantitativa }\end{array}$ & $\begin{array}{l}\text { Psychology/Psicologia } \\
\text { Reflexão e Crítica }\end{array}$ \\
\hline 2 & Oliveira RF, et al. (2017) & $\begin{array}{l}\text { Incidência da síndrome de burnout } \\
\text { nos profissionais de enfermagem: } \\
\text { uma revisão integrativa }\end{array}$ & $\begin{array}{c}\text { Explorar as produções científicas disponíveis } \\
\text { analisando a incidência da Síndrome de Burnout nos } \\
\text { profissionais da área da enfermagem }\end{array}$ & Estudo Descritivo & $\begin{array}{l}\text { Revista de Enfermagem do } \\
\text { Centro-Oeste Mineiro }\end{array}$ \\
\hline 3 & Mourão AL, et al. (2017) & $\begin{array}{c}\text { Síndrome de burnout no contexto da } \\
\text { enfermagem }\end{array}$ & $\begin{array}{l}\text { Abordar as experiências inerentes ao contexto dos } \\
\text { profissionais da enfermagem e suas possíveis relações } \\
\text { com a síndrome de burnout }\end{array}$ & Descritivo exploratório & $\begin{array}{l}\text { Revista Baiana de Saúde } \\
\text { Pública }\end{array}$ \\
\hline 4 & Santos JLG, et al. (2019) & $\begin{array}{l}\text { Síndrome de burnout entre } \\
\text { enfermeiros de um hospital } \\
\text { universitário }\end{array}$ & $\begin{array}{l}\text { Identificar os níveis da síndrome de burnout entre } \\
\text { enfermeiros de um hospital universitário. }\end{array}$ & Estudo transversal & $\begin{array}{l}\text { Revista Baiana de } \\
\text { Enfermagem }\end{array}$ \\
\hline 5 & Merces MC, et al. (2017) & $\begin{array}{l}\text { Prevalência da Síndrome de Burnout } \\
\text { em profissionais de enfermagem da } \\
\text { atenção básica à saúde }\end{array}$ & $\begin{array}{l}\text { Estimar a prevalência da Síndrome de Burnout entre } \\
\text { profissionais de enfermagem da Atenção Básica à } \\
\text { Saúde de um município do sudoeste baiano. }\end{array}$ & Estudo transversal & Rev Fund Care Online \\
\hline 6 & Magalhães BC, et al. (2020) & $\begin{array}{c}\text { Síndrome de burnout em uma } \\
\text { unidade hospitalar: Percepções da } \\
\text { equipe de enfermagem }\end{array}$ & $\begin{array}{l}\text { Conhecer a percepção da equipe de enfermagem } \\
\text { acerca da Síndrome de Burnout. }\end{array}$ & $\begin{array}{l}\text { Descritivo e } \\
\text { exploratório }\end{array}$ & $\begin{array}{l}\text { Revista Online de } \\
\text { Pesquisa: Cuidado é } \\
\text { Fundamental }\end{array}$ \\
\hline 7 & Silva AR, et al. (2020) & $\begin{array}{l}\text { Processo de trabalho hospitalar e a } \\
\text { síndrome de Burnout em profissionais } \\
\text { de enfermagem }\end{array}$ & $\begin{array}{c}\text { Identificar os fatores do processo de trabalho que } \\
\text { favorecem o desenvolvimento da Síndrome de Burnout } \\
\text { em profissionais de enfermagem na assistência } \\
\text { hospitalar. }\end{array}$ & Estudo Descritivo & $\begin{array}{l}\text { Revista Online de } \\
\text { Pesquisa: Cuidado é } \\
\text { Fundamental }\end{array}$ \\
\hline 8 & Paiva JDM, et al. (2019) & $\begin{array}{l}\text { Fatores desencadeantes da síndrome } \\
\text { de burnout em enfermeiros }\end{array}$ & $\begin{array}{c}\text { Identificar o conhecimento exposto na literatura sobre } \\
\text { os fatores desencadeantes da Síndrome de Burnout } \\
\text { em enfermeiros. }\end{array}$ & Estudo Descritivo & Rev enferm UFPE online \\
\hline 9 & Costa SMS, et al. (2020) & $\begin{array}{l}\text { Síndrome de burnout em profissionais } \\
\text { de enfermagem }\end{array}$ & $\begin{array}{c}\text { Identificar a prevalência da Síndrome de Burnout em } \\
\text { profissionais de Enfermagem de um hospital de } \\
\text { emergência }\end{array}$ & Estudo transversal & Rev enferm UFPE online \\
\hline 10 & Vidotti V, et al. (2018) & $\begin{array}{l}\text { Síndrome de Burnout e o trabalho em } \\
\text { turnos na equipe de enfermagem }\end{array}$ & $\begin{array}{l}\text { Analisar os fatores associados à síndrome de Burnout, } \\
\text { segundo o turno de trabalho da equipe de enfermagem. }\end{array}$ & Estudo transversal & $\begin{array}{l}\text { Rev. Latino-Am. } \\
\text { Enfermagem }\end{array}$ \\
\hline 11 & Moreira AS, et al. (2020) & $\begin{array}{l}\text { Fatores psicossociais e Síndrome de } \\
\text { Burnout entre os profissionais dos } \\
\text { serviços de saúde mental }\end{array}$ & $\begin{array}{l}\text { Identificar os fatores biopsicossociais no trabalho } \\
\text { associados à Síndrome de Burnout em profissionais da } \\
\text { saúde mental }\end{array}$ & Estudo transversal & $\begin{array}{l}\text { Rev. Latino-Am. } \\
\text { Enfermagem }\end{array}$ \\
\hline
\end{tabular}

Fonte: Barbosa IEB, et al., 2021. 


\section{DISCUSSÃO}

\section{Incidência da SB nos profissionais de Enfermagem}

O estudo de Campos IC, et al. (2015), investigou a prevalência da SB nos profissionais de enfermagem, realizando uma comparação entre os enfermeiros, técnicos e auxiliares de enfermagem, a pesquisa foi composta por 166 profissionais. Utilizando o questionário sociodemográfico e ocupacional em conjunto com o Inventário de Burnout de Maslach, observou-se (57\%) da prevalência da SB nos trabalhadores.

Na pesquisa Merces MC, et al. (2017), verificou a prevalência da SB em profissionais de enfermagem de uma Atenção Básica de Saúde, o estudo foi composto por 60 profissionais de enfermagem. Utilizou-se também o questionário sociodemográfico e ocupacional em conjunto com o Inventário de Burnout de Maslach, após a tabulação dos dados, a prevalência da SB foi de (58\%) de acordo com os critérios escolhidos pelos pesquisadores.

Dois dos estudos selecionados para revisão, que buscaram investigar a prevalência da SB em profissionais de enfermagem, apresentaram alta prevalência nos trabalhadores, sendo os auxiliares de enfermagem com maior prevalência (52,94\%), (50\%) nos técnicos de enfermagem e (32\%) em enfermeiros (CAMPOS IC, et al., 2015).

\section{Principais sinais aparentes da SB no trabalho}

Depois de muitos componentes iniciados da síndrome, são encontrados no campo do emprego: perda de autonomia, mudanças organizacionais frequentes, questões de parceria com colegas, comunicação ineficiente, nenhum reconhecimento e apreciação, tarefas desnecessárias a serem realizadas em pouco tempo e com um número limitado de pessoa, trabalho repetitivo que favorece posturas inadequada (CAMPOS IC, et al., 2015).

Além dos desafios organizacionais que os enfermeiros enfrentam, há os aspectos físicos, espirituais, sociais e psicológicos da responsabilização de seus pacientes e também a realidade de que dentro de uma instituição, a enfermagem não obtém prestígio. Além disso, é introduzida uma estreita interação com o desconforto, a miséria e a mortalidade, fazendo com que o profissional de enfermagem precise ter muito controle emocional diante de situações delicadas. O hospital do dia-a-dia, que faz com que os profissionais atinjam a fadiga e se tornem frios, desmotivados, apáticos e cansados, permanece nesse sentido (MOREIRA AS, et al., 2020).

\section{Condições trabalhistas da enfermagem relacionadas à SB}

Ambas as variáveis ocupacionais e sociais do paciente são propícias à causa da patologia física e emocional, absenteísmo, lesões no trabalho, erros de medicamentos, estresse, sobrecarga de emprego e perda de lazer. Quaisquer potenciais razões agravantes para o adoecimento são aplicadas a isso, como período de preparação limitado, mais de 30 horas de carga horária semanal, trabalho duplo, menos de um ano de trabalho e função gerencial (VIDOTTI V, et al., 2018).

Diante do levantamento realizado, observa-se um grande sentimento de desamparo para a equipe de enfermagem, particularmente àqueles que trabalham em um setor onde há um grande risco de morte. A relação é inevitável ao lado de um paciente e profissional de saúde, pois o paciente continua no hospital por um período prolongado de tempo, e o profissional ainda se entristece dessa falha, pois há o processo de morte. Reconhece-se que o luto e a miséria não vividos do profissional, juntamente com um convívio avassalador no ambiente de trabalho, são variáveis favoráveis para o aparecimento da Síndrome de Burnout (OLIVEIRA RF, et al., 2017).

\section{Fatores que contribuem para a SB na equipe de enfermagem}

Segundo Costa SMS, et al. (2020) existem várias causas que contribuem para o adoecimento do profissional, especialmente em Unidades de Terapia Intensiva (UTIs), tais como: não receber valorização e ajuda no trabalho; sobrecarga de trabalho; dificuldades de interação de liderança; operando o turno da noite; 
crise ética entre crenças e questões profissionais; dificuldades para lidar com a mortalidade e rigidez burocrática. Qualquer uma dessas condições pode induzir efeitos físicos, tais como: irritabilidade; envelhecimento prematuro; uso de drogas; dor de cabeça e enxaqueca; azia; fadiga física e mental; náusea; taquicardia; ofegante; dor lombar; pernas; dificuldade de alimentação; insônia; sonhos de trabalho e não ser capaz de descansar; sintomas que eventualmente afetam o bem-estar do trabalhador.

Por outro lado, existem muitas causas diferentes que podem desencadear a doença para o trabalho de enfermagem em uma unidade básica de saúde, tais como: número inadequado de funcionários; tarefas rotineiras; ritmo de trabalho rápido; perda de motivação; falta de respeito e reconhecimento; usuários agressivos; insegurança; ameaças; morte de pacientes; mau tempo no trabalho e conflitos de consumo; e fadiga emocional devido à estreita conexão gerada pela proximidade. Além dos estresses organizacionais e das interações cotidianas vividas, todas essas variáveis criam uma grande sobrecarga emocional que pode afetar a interação entre paciente e especialista (MOURÃO AL, et al., 2017).

Um forte candidato ao desenvolvimento da síndrome de burnout é o técnico de enfermagem, onde os desafios em distinguir posições entre enfermeiros, técnicos e auxiliares de enfermagem destaca a realidade de que o técnico é o especialista menos independente no local de trabalho, além de ser o profissional que normalmente passa mais tempo com o paciente e experimenta desconforto e angústia todos os dias (PAIVA JDM, et al., 2019).

Há uma prova muito boa de que os profissionais de enfermagem têm uma grande chance de não ter Síndrome de Burnout em relação a profissionais que não têm esposa e filhos, mesmo com horário de trabalho duplo e casas organizadas, onde acabam não cumprindo a posição de maternidade e paternidade. Isso pode estar correlacionado com profissionais que têm filhos que, portanto, têm mais comprometimento, experiência, estabilidade e aspirações mais razoáveis (SANTOS JLG, et al., 2019).

\section{Variáveis sociodemográficas e ocupacionais da SB na equipe de enfermagem}

Os profissionais de enfermagem estão bastante expostos à experiência das famílias no paradigma da Política de Saúde da Família, que é definida pela prevenção de doenças e promoção da saúde por meio de ações educativas realizadas em casa e, portanto, em risco, inseguras e propícias aos riscos à saúde. Essas considerações aplicadas à falta de serviços para atender às diversas necessidades que enfrentam a realidade e as deficiências na rede de atenção à saúde acabam impactando na capacidade de esforços para enfrentar desafios e a realização profissional (MAGALHÃES BC, et al., 2020)

Fatores como aspectos da organização e do processo de trabalho, viés e presença de mortalidade que tanto acontece são vistos como fontes de tensão e fadiga psicológica. Observar o profissional de saúde que lida de perto com doenças infecciosas como o HIV, pois há também uma escassez de cura para a doença, aliado a um número muito alto de casos afetados, resultando em agressividade e raiva de pacientes e familiares (SILVA AR, et al., 2020).

A incidência da síndrome é mais evidente em jovens funcionários que ainda não chegaram aos 30 anos, onde fatores como falta de experiência que desencadeia incerteza ou choque de verdade, medo do desemprego e, por vezes, fadiga física e psicológica subestimada podem contribuir. Nota-se que quanto mais tempo o período de trabalho, menos perigoso é para o bem-estar do enfermeiro, que ao longo dos anos já acumulou uma bagagem substancial de conhecimentos e habilidades para lidar com questões de saúde e, portanto, seu reconhecimento profissional já é desenvolvido e aceito (MOURÃO AL, et al., 2017).

Segundo Merces MC, et al. (2017) um conhecimento minucioso e dedicado dos padrões de vida do trabalho, que abrange elementos de bem-estar, proteção física, emocional e social, é o padrão de vida no trabalho. Depende não apenas de uma parte, ou seja, depende do indivíduo e da organização ao mesmo tempo. A necessidade de comprometimento das organizações com a situação de saúde dos trabalhadores é vista por uma estratégia adequada de recursos humanos e de gestão, especialmente levando em conta os colaboradores que possuem maior proximidade físico-psicológica com o paciente/família, a fim de prevenir doenças e preservar a consistência do serviço prestado. 


\section{CONSIDERAÇÕES FINAIS}

Em relação à questão profissional, a dupla jornada de trabalho foi um fator significativo correlacionado com a síndrome de burnout em todas as suas dimensões. Deve-se lembrar que, além disso, enfermeiros, auxiliares e técnicos de enfermagem estavam entre os examinados por serem extremamente suscetíveis à síndrome. Diante do exposto, é clara a necessidade de prestar atenção ao bem-estar emocional dos profissionais da enfermagem, bem como ao perfil sociodemográfico e ocupacional daqueles com maior risco de desenvolvimento do agravo em estudo. Logo, realizar acompanhamento psicológico com esses profissionais de saúde é algo extremamente válido, valorizando assim a sua saúde mental, com a finalidade de prevenir complicações futuras relacionadas a SB.

\section{REFERÊNCIAS}

1. ALMEIDA LY, et. al. Avaliação do apoio social e estresse em estudantes de enfermagem. Rev Esc Enferm USP, 2018; 52: e03404. Vol.12(10) | e4383

2. BARROCAS PHN, et al. Condições de trabalho de técnicas de enfermagem de um hospital particular. O público e o privado, 2015; no 25: 77-100.

3. BATISTA KO, et al. Síndrome de burnout em enfermeiros: consequências na atividade profissional. ReBIS, 2019; 1(4): 6165.

4. CAMPOS ICM, et al. (2015). Fatores Sociodemográfi cos e Ocupacionais Associados à Síndrome de Burnout em Profi ssionais de Enfermagem.

5. COSTA SMS, et al. Síndrome de Burnout em profissionais de enfermagem. Rev enferm UFPE on line. 2020;14: e243351.

6. CRUZ SP, ABELLÁN MV. Desgaste profissional, stress e satisfação no trabalho do pessoal de enfermagem em um hospital universitário. Rev. Latino-Am. Enfermagem, 2015; 23(3): 543-552.

7. LAGE CEB, ALVES MS. (Des) valorização da enfermagem: implicações no cotidiano do enfermeiro. Enferm. Foco, 2016; 7(3/4): 12-16.

8. LOIOLA E, MARTINS MC. Autoeficácia no trabalho e síndrome de burnout em profissionais de enfermagem. Psicologia, Saúde \& Doenças, 2019; 20(3): 813-823.

9. LOPES LS, et al. Yoga para Manejo e Redução da Síndrome de Burnout em Profissionais da Saúde. Rev Bras Terap e Saúde, 2019; 9(2): 1-7.

10. MAGALHÃES BC, et al. Síndrome de Burnout em uma unidade hospitalar: percepções da equipe de enfermagem. 2020 jan/dez; 12:1004-1010.

11. MERCES MC, et al. Prevalence of Burnout Syndrome in nursing professionals of basic health care. Rev Fund Care Online. $2017 \mathrm{jan} / \mathrm{mar} ; 9(1): 208-214$.

12. MORENO JK, et al. Síndrome de burnout e fatores de estresse em enfermeiros nefrologistas. Rev Enferm UFPE, 2018; 12(4): 865-871.

13. MOURA RS, et al. Estresse, burnout e depressão nos auxiliares e técnicos em enfermagem das unidades de terapia intensiva. Enfermería global, 2019; no 54: 94-108.

14. MOREIRA AS, et al, Psychosocial factors and Burnout Syndrome among mental health professionals. Rev. Latino-Am. Enfermagem. 2020;28: e3336.

15. MOTA BS et al. As contribuições da síndrome de burnout para o déficit do trabalho da enfermagem: revisão integrativa da literatura. Revista Eletrônica Acervo Saúde, 2020; 12(10): e4383.

16. NOBRE DFR, et al. Avaliação do burnout em enfermeiros de um serviço de urgência geral. Rev Bras Enferm, 2019; 72(6): 1533-9.

17. OLIVEIRA RF, et al. Incidência da Síndrome de Burnout nos Profissionais de Enfermagem: Uma Revisão Integrativa. Revista de Enfermagem do Centro-Oeste Mineiro. 2017; 7: e1383.

18. PEREIRA MMA, GOMES ARS. Stress, burnout e avaliação cognitiva: estudo na classe de enfermagem. Arquivos Brasileiros de Psicologia, 2016; 68(1): 72-83.

19. RAMOS CEB, et al. Impactos da síndrome de burnout na qualidade de vida dos profissionais de enfermagem da atenção básica à saúde. R bras ci Saúde, 2019; 23(3): 285-296.

20. RIVAS E, BARRAZA-MACÍAS A. Síndrome de Burnout no pessoal de enfermagem e sua relação com quatro variáveis laborais. Enfermería Universitaria, 2018; 15(2): 136-146.

21. SANCLEMENTE-VINUE I, et al. A voz da enfermagem como ferramenta para promover o engagement no trabalho. Rev. Latino-Am. Enfermagem, 2019; 27: e3208.

22. SANTOS JLG, et al. Síndrome de burnout entre enfermeiros de um hospital universitário. Rev baiana enferm. 2019;33: e29057.

23. SILVA AR, et al. Processo de trabalho hospitalar e a Síndrome de Burnout em profissionais de enfermagem. 2020 jan/dez; 12:921-927.

24. SILVA FG, et al. Predisposição para síndrome de burnout na equipe de enfermagem do serviço de atendimento móvel de urgência. Enferm. Foco, 2019; 10(1): 40-45.

25. SILVA-JUNIOR RF, et al. Personalidade hardiness e fatores associados em profissionais da saúde atuantes em serviços que atendem pacientes críticos. Ciência \& Saúde Coletiva, 2020;

26. SOUSA LMM, et al. A metodologia de revisão integrativa da literatura em enfermagem. Revista Investigação em Enfermagem, 2017; 17-26.

27. VALERETTO FA, ALVES DF. Fatores desencadeantes do estresse ocupacional e da síndrome de burnout em enfermeiros. Revista Saúde Física \& Mental, UNIABEU. 2013;

28. VASCONCELOS EM, MARTINO MMF. Preditores da síndrome de burnout em enfermeiros de unidade de terapia intensiva. Rev Gaúcha Enferm., 2017; 38(4): e65354.

29. VIDOTTI V, et al. Burnout Syndrome and shift work among the nursing staff. Rev. Latino-Am. Enfermagem. $2018 ; 26$ : e3022.

30. VIEIRA I, RUSSO J. Burnout e estresse: entre medicalização e psicologização. Revista Saúde Coletiva, 2019; 29(2): e290206. 